\title{
Modelling the interfacial flow of two immiscible liquids in mixing processes
}

\author{
H. Tang ${ }^{1}$ and L.C. Wrobel ${ }^{2}$

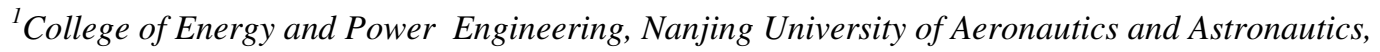 \\ Jiangsu 210016, China \\ ${ }^{2}$ School of Engineering and Design, Brunel University, Uxbridge, Middlesex, UB8 3PH, UK
}

\begin{abstract}
This paper presents an interface tracking method for modelling the flow of immiscible metallic liquids in mixing processes. The methodology can provide an insight into mixing processes for studying the fundamental morphology development mechanisms for immiscible interfaces. The volume-of-fluid (VOF) method is adopted in the present study, following a review of various modelling approaches for immiscible fluid systems. The VOF method employed here utilises the piecewise linear for interface construction scheme as well as the continuum surface force algorithm for surface force modelling. A model coupling numerical and experimental data is established. The main flow features in the mixing process are investigated. It is observed that the mixing of immiscible metallic liquids is strongly influenced by the viscosity of the system, shear forces and turbulence. The numerical results show good qualitative agreement with experimental results, and are useful for optimisating the design of mixing casting processes.
\end{abstract}

Key words: immiscible alloys, mixing, VOF, shear flow, interface tracking 


\section{Introduction}

Casting immiscible engineering alloys is a challenging problem due to metallic and hydrodynamic phenomena which take place during the process; various research efforts are still in progress to develop a process methodology for obtaining a fine and uniformly dispersed microstructure. Evidence shows that the liquid-liquid decomposition and spatial behaviour in the molten state dominate the solidified microstructure of immiscible alloys. However, the hydrodynamic behaviour in the molten state is not well understood. Experiments encounter some difficulties to provide a complete insight into the process. Therefore, a computational method has been developed to investigate the hydrodynamic behaviour of immiscible metallic alloys in a novel casting process called rheomixing, based on the use of a twin-screw extruder (TSE).

\section{Process of microstructure evolution}

A rheomixing process was developed based on previous experience in the processing of semisolid metal (SSM) slurry by a TSE [1]. TSE is commonly used in polymer processing [2,3], and provides sufficient shear flow in the rheomixing process to create a fine and homogeneous droplet dispersion, and enough viscous force in the semisolid state to counterbalance the Stokes and Marangoni motions. However, there are still some limitations for the experiments to provide an insight into the flow during the process.

Applications of TSE in immiscible engineering alloy processing generate different flow mechanisms, which results in the desired solidified microstructure. The study of immiscible binary alloys in shear-induced turbulent flows is important to provide more detailed information into the rheomixing process, to increase our control of the rheology of an emulsion and its solidified microstructure. An understanding of the fluid dynamics inside and around a suspended drop is necessary for delineating the mechanisms of microscopic transport and microstructure of immiscible alloys.

\subsection{Rheological behaviour}

A large number of metallic binaries exhibit limited mutual component solubility in the liquid state. These alloys, commonly named monotectic alloys, are of special technical 
importance and became the subject of intensive studies during the last decades. Owing to the difficulties of high-temperature experimentation and the absence of a generally accepted measuring technique, only a few binaries have been sufficiently studied in the miscibility gap region at elevated temperature. The process of decomposition of the homogeneous melt at high temperature into two immiscible melts, which are generally of different density, is very rapid and prevents the production of finely dispersed mixtures with the prospect of practical application. A decomposition phenomenon is caused by processes like gravity-dependent sedimentation, thermocapillary convection, etc.

Alloys with a miscibility gap have been studied under microgravity conditions since the 1970 s by NASA $[4,5]$, to investigate the processing of technologically interesting materials, such as $\mathrm{Al}-\mathrm{Pb}$ for bearings or $\mathrm{Mn}-\mathrm{Bi}$ for magnetic applications. Microgravity experiments and containerless processing offer new possibilities to investigate the properties of such alloys. However, in microgravity, it is found that a fine dispersion of the minority phase cannot be formed due to Marangoni motion, which causes a coarse phase separation on Earth. Attempts to overcome these problems never gave satisfactory results.

Solidification in the casting process is largely determined by thermodynamic and kinetic factors. From the kinetic point of view, solidification results in an abrupt decrease of atomic mobility. Since the raw material is initially in the molten state, the heat release is large, which means that large amounts of heat have to be extracted quickly. The microstructure of the cast product (i.e. secondary-phase particle distribution parameters, such as secondary dendrite arm spacing, particle and/or eutectic colony size, matrix composition, etc.) is mostly determined by both phase diagram equilibrium and kinetic considerations, in particular the liquid composition and casting procedure. The nature of the process in the liquid state is described in the next section.

\subsection{Dominating processes}

The transformation or decomposition process does not occur instantaneously. Generally, the second phase is first nucleated as very fine droplets, which grow by diffusion and can also settle due to the action of gravity, leading finally to a 
macroscopically layered structure of the fluids. All three processes - nucleation, growth and sedimentation - occur concurrently. Possible factors affecting phase segregation could be as follows:

- Nucleation of the minority phase

- Growth of the nuclei-diffusion-convection

- Agglomeration due to Brownian motion

- Sedimentation due to gravity

- Coagulation due to collision

- Interfacial energy

- Ostwald ripening or coarsening

- Marangoni migration due to thermocapillary forces

- Fraction of the minority phase and cooling rate

- Potential flow due to the morphology of the solidified front

However, the dominating factors will be different in different processes. For example, under microgravity process, Marangoni motion is the main factor influencing the microstructure of solidification rather than Stokes motion under normal gravity process. However, Marangoni motion is still a dominating factor in orientating temperature gradient process under normal gravity [6]. Features of immiscible liquid alloys are summarised in Table 1.

Table 1. Features of immiscible liquid alloys

\begin{tabular}{|c|c|c|}
\hline Main processes & Main features & Results \\
\hline Complex mixture & Atoms mixing, Brownian motion & $\begin{array}{l}\text { one phase liquid } \\
\text { forming fine drops, size<one }\end{array}$ \\
\hline Nucleation & Gibbs free energy, temperature gradients & micron \\
\hline Growth & Diffusion, temperature gradients & coarsening, size $<$ microns \\
\hline Sedimentation & Stokes motion & stratification \\
\hline Migration & Marangoni motion & segregation \\
\hline Collision/coagulation & Stokes/Marangoni motion & agglomeration \\
\hline
\end{tabular}




\section{Mixing process}

Mixing immiscible fluids have been studied experimentally and numerically. A number of experimental processes and industrial applications have been invented for casting immiscible alloys since the 1970s; some basic concept of mixing approaches and a brief comparison of approaches for casting immiscible alloys are described in this section.

\subsection{Mixing immiscible fluids}

Two types of flows are important to study the fundamental mechanisms of mixing processes: simple shear flow and pure elongational flow. Pure elongational flow is the most efficient to break up fluid particles. However, it is impossible to achieve pure elongational flow in the confined flow geometries encountered in mixing machines and during limited periods of time. So, in practice, shear flow is more important. A shearing flow at its beginning effectively increases the distance between two neighbouring particles, but the efficiency decreases with time. As a consequence, a single-screw extruder (SSE) with continuous channel geometry is a poor mixing device. If the shearing flow is periodically reoriented, the mixing efficiency is significantly enhanced [7]. Therefore, TSE is a better mixing device because materials are reoriented due to the transferring from one screw channel to the adjacent one in the conveying zone of a corotating twin-screw extruder. However, for casting immiscible alloys, the most important process after intensive and distribution processes is the generation of a stable suspension for solidifying the homogeneous dispersion of fluid particles.

\subsection{Rheomixing process by TSE}

Rheomixing is an integrated process for production of near-net shape components from immiscible alloys, such as $\mathrm{Zn}-\mathrm{Pb}, \mathrm{Al}-\mathrm{Pb}, \mathrm{Ga}-\mathrm{Pb}$ systems. Rheomixing starts from melting metal ingots with predetermined compositions and pouring the melts into the rheomixer above the miscibility gap. Molten metal is then sheared through the miscibility gap to form a uniformly mixed paste, and transferred into a shot sleeve. This is subsequently injected into a die. 
Understanding and simulating the flow around the twin screws has always been a difficult and challenging task due to the complex geometry in the vicinity of the interscrew regions and the large deformations induced by the two rotating screws.

The flow fields of a self-wiping TSE can be divided into two hypothetical regions, one in which the two screws intermesh (nip or intermeshing region) and the other far away from the nip, referred to as the convey region. The flow in the convey region is considered to be similar to the flow in the channel of a SSE. The advantages of a TSE over a SSE are often attributed to the nip region, which is considered to have a positive displacement character [8].

The flow field of the intermeshing co-rotating twin-screw extruder undergoes cyclic stretching, folding, and reorienting [9]. Basically, the main feature of a twin-screw extruder is a strong shear flow field produced by co-rotating intermeshing screws. Understanding the fundamental flow mechanisms on a microstructural scale will help to optimise the mixing processes. However, flow mechanism studies are normally carried out for laminar viscoelastic flows of highly viscous materials in polymer processing. No studies have been reported for immiscible liquid alloys and semi-solid metal alloys until TSE was employed for casting processes [10].

A description of classifications and configurations is given in [11]. The basic elements of the twin-screw extruder are the screws and mixing blocks, such as the kneading discs. The elements of the screw, with suitable numbers of mixing blocks, are assembled together inside the barrel in order to reach the best mixing efficiency within the duration of the process.

\section{Modelling approaches}

The solidified microstructure of immiscible alloys strongly depends on processing conditions, especially the liquid-liquid decomposition and spatial separation behaviour within the molten state. There have been several attempts to model at least parts of the microstructure evolution in the liquid state or even the whole process from nucleation until the formation of layers. Two approaches were described in a review paper on immiscible liquid alloys [12]. However, there are more numerical approaches for

multiphase flows available to simulate possible phase segregation, coagulation, 
collision, immiscible interface, sedimentation, etc. These include the dissipative particle dynamics (DPD) approach for the phase segregation of binary systems at the mesoscale, the Euler-Euler approach for macrostructural modelling, various immiscible interface tracking approaches for both microstructural and macrostructural modelling, molecular dynamics (MD) approaches for the simulation in the atomistic scale, as well as combined approaches. A brief summary is given below.

\subsection{Various numerical methods}

\subsubsection{Population dynamics approach}

The first method based on population dynamics (PD) was the Smoluchowiski approach [13], developed originally in 1916 for an initially monosized dispersion and extended in 1926 to a polydisperse system for arbitrary kinds of particle/droplet motions. This approach has been studied for decades in the fields of aerosol mechanics and cloud physics to understand the coagulation of droplets, and has also been used to solve the problem of droplet size evolution during decomposition of immiscible liquid including immiscible alloys. The Smoluchowiski equation is given by:

$$
\frac{\partial n}{\partial t}=\frac{1}{2} \int_{0}^{v} K\left(v, v-v^{\prime}\right) n(v, t) n\left(v-v^{\prime}, t\right) d v^{\prime}-n(v, t) \int_{0}^{\infty} n\left(v^{\prime}, t\right) K\left(v, v^{\prime}\right) d v^{\prime}
$$

To include effects such as sedimentation, nucleation, growth and coagulation in the same mean-field approach, Eq. (1) is redefined as:

$$
\begin{aligned}
& \frac{\partial n}{\partial t}+\nabla\left(U(x, y, z) n(x, y, z)+\frac{\partial}{\partial R}\left(\frac{d R}{d t} \cdot n\right)=\right. \\
& I(x, y, z)+\frac{1}{2} \int_{0}^{v} K\left(v, v-v^{\prime}\right) n(v, t) n\left(v-v^{\prime}, t\right) d v^{\prime}-n(v, t) \int_{0}^{\infty} n\left(v^{\prime}, t\right) K\left(v, v^{\prime}\right) d v^{\prime}
\end{aligned}
$$

where $K\left(v, v^{\prime}\right)$ - reaction rate, $v, v^{\prime}$ - droplet volume, $n(v, t)$ - density of drops of volume $v, v+\mathrm{d} v$ at time $t, U(x, y, z)$ - droplet velocity, $n(x, y, z)$ - given distance of spatial evolution, $I(x, y, z)$ - nucleation rate.

To solve this equation, special assumptions are employed and treated analytically or numerically to model the structure evolution in dispersions. In the field of immiscible liquid alloys, Rogers and Davis [14], Ratke et al. [15,16] used Eq. (1) to predict droplet size evolution either under Earth or microgravity conditions, for $\mathrm{Zn}-\mathrm{Bi}$ or $\mathrm{Zn}-\mathrm{Pb}$ alloys 
without considerations of nucleation and growth. Alkemper and Ratke [17] solved Eq. (2) (neglecting coagulation) to describe the solidified microstructure of Al-Bi samples. Carberg and Fredriksson [18], Bergmon and Fredriksson [19] analysed the droplet-size evolution in $\mathrm{Zn}-\mathrm{Bi}$ and $\mathrm{Zn}-\mathrm{Pb}$, respectively, by only considering growth under microgravity condition. Ahlborn et al. [20] analysed the segregation behaviour of rapidly cooled monotectic $\mathrm{Al}-\mathrm{In}$ and $\mathrm{Al}-\mathrm{Pb}$ alloys, obtaining good agreement with experimental results by considering growth and Maragoni motion rather than only growth. Recent contributions were presented by Zhao et al. [6,21,22] but collision and coagulation were still neglected. Thus, applications of PD approaches are still limited in the field of immiscible alloys. In the solution of Eq. (1), various Monte Carlo (MC) techniques have been developed for the growth of dispersed systems and generally fall into constant-number or constant-volume methods, such as the hybrid Monte Carlo method for simulating two-component aerosol coagulation and phase segregation [23].

\subsubsection{Discrete multi-particle approach}

An alternative method is the discrete multi-particle (DMP) approach developed by Ratke and Diefenbach [24,25]. The approach starts by setting up a three-dimensional sample and imposing a temperature profile which is either calculated or known experimentally, rather than solving a complicated set of partial differential equations or integro-differential equations. Then, the sample is divided into thin slices or cubes and the thermodynamic theory of solidification is adopted to calculate nucleation and growth of droplets, as well as Ostwald ripening. A concentration difference arises between slices, and Fick's law of diffusion is employed to describe the general diffusion of the solute. Positions and coagulations of droplets are tracked and calculated until either a sample two-layer structure is obtained or a locally varying distribution of drops in a solidified sample. This approach was tested experimentally, but theoretical predictions and experimental results disagreed considerably. Therefore, implementations of this approach are rarely found in the literature. 


\subsubsection{Dissipative particle dynamics approach}

Dissipative particle dynamics (DPD) is another discrete particle approach based on employing equations from kinetic theory. Kinetics governs the rapid quenching of fluid from the high-temperature, single-phase region to the two-phase region. The growth kinetics of binary immiscible fluid and phase separation in these systems has been studied by using a variety of theoretical and computational tools [26]. These are lattice gas automata [27], molecular dynamics [28], continuum models based on Langevin equations [29] and dissipative particle dynamics [26,30]. DPD is used to simulate phase segregation and fluid mixing in binary systems. The growth kinetics represented by the temporal increase of average cluster size for both colloid aggregates and binary immiscible mixtures can be controlled by the scaling factors of the collision operator. The mesoscopic character of the DPD method, employed for simulating phase separation, enables the observation of the two scaling regimes in the course of a single DPD simulation. By increasing the value of differential pressures, one can easily control the surface tension in the particle system, studying the broad spectrum of the immiscible binary mixtures, from Brownian-dominated systems to stable emulsion-like substances. However, this method is limited to simulate possible phase segregation of immiscible alloys without considering forced convection and enhanced turbulent flow. Obviously, it is not a suitable approach for the present study.

There are still some well known approaches besides the phase segregation approaches listed in the above paragraph, such as the Cahn-Hilliard $(\mathrm{C}-\mathrm{H})$ model, the phase field model, the Novick-Cohen-Pego model, which are applied for phase separation of metallic systems although they are not well developed for industrial applications [31]. Kupper and Masbaum [32] used a C-H model to analyse growth and Ostwald ripening, and results are better than those predicted by the LSW theory [33].

\subsubsection{Summary and comparison}

As described in the previous sections, several efforts have been made to understand the processing of immiscible alloys by simulation approaches. However, there is no approach that can cover all factors listed in Table 1 , from regions above $T_{\mathrm{c}}$ down to $T_{\mathrm{m}}$ in the phase diagram. Each approach can predict some of the factors while neglecting 
others. Most approaches have been developed for immiscible fluids in aerosol and other chemical areas, and further developments are needed to match the physical features of immiscible liquid alloys. Generally speaking, these approaches are powerful techniques to predict the decomposition and microstructural evolution, if the real thermophysical properties are known with enough accuracy, or alternatively, these approaches can be used for design tests if these properties are known accurately. Unfortunately, there is obviously a lack of knowledge on immiscible alloys, since interfacial tension, viscosity, diffusion coefficients are functions of composition, temperature, and shear rate for semisolid metals and not know with sufficient accuracy. A brief summary and comparison of modelling approaches is given in Table 2.

\subsection{VOF interface tracking method}

The previously described methods are normally used for the modelling of microstructural evolution during conventional solidification and to simulate the nature of phase change. However, rheomixing is a process to mix two segregated liquid phases, and involves forced convection and enhanced turbulent flow by imposing shear forces. Thus, the hydrodynamic behaviour is the dominating feature, which strongly affects the solidified microstructure. A suitable simulation approach is required for studying the fundamental mechanism of shear-induced turbulent flow in immiscible liquid alloy systems. Advances in computational fluid mechanics have provided the basis for further insight into the dynamics of immiscible alloy flows. The Euler-Euler approach is the most popular method, where the different phases are treated mathematically as interpenetrating continua. Since the volume of a phase cannot be occupied by other phases, the concept of phase volume fraction is introduced. These volume fractions are assumed to be continuous functions of space and time, and their sum is equal to one. Conservation equations for each phase are derived to obtain a set of equations, which have a similar structure for all phases. These equations are closed by providing constitutive relations that are obtained from empirical information or, in the case of granular flows, by application of kinetic theory. Several different Eulerian multiphase models are available, the VOF model being the most successful approach to capture mixing, merging, folding and stretching of a two-fluid system under an imposed force. 
Table 2

Summary and comparison of possible modelling approaches for simulation of immiscible liquid alloys [13-31]

\begin{tabular}{|c|c|c|c|c|}
\hline & Approaches & States & Areas & Limitations \\
\hline \multicolumn{2}{|c|}{ Population dynamics } & $\begin{array}{l}\text { well developed theoretically, } \\
\text { describes all factors of phase } \\
\text { segregation, main approach for } \\
\text { studying the nature of } \\
\text { immiscible fluids }\end{array}$ & $\begin{array}{l}\text { immiscible } \\
\text { liquids and } \\
\text { alloys }\end{array}$ & $\begin{array}{l}\text { too complicated } \\
\text { and no solution w/o } \\
\text { simplifying, no } \\
\text { fluid dynamics }\end{array}$ \\
\hline \multicolumn{2}{|c|}{ Discrete multi-particle } & $\begin{array}{l}\text { possibly include all factors, } \\
\text { easier to implement without } \\
\text { solving PDE }\end{array}$ & $\begin{array}{l}\text { immiscible } \\
\text { alloys }\end{array}$ & $\begin{array}{l}\text { poor agreement } \\
\text { with experiments }\end{array}$ \\
\hline \multirow{4}{*}{ 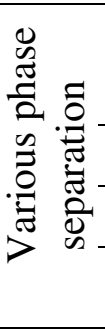 } & $\begin{array}{l}\text { Dissipative particle } \\
\text { dynamics }\end{array}$ & $\begin{array}{l}\text { based on kinetic theory of phase } \\
\text { separation, controlled by } \\
\text { pressure difference }\end{array}$ & $\begin{array}{l}\text { immiscible } \\
\text { liquids }\end{array}$ & $\begin{array}{l}\text { no fluid dynamics, } \\
\text { mesoscale }\end{array}$ \\
\hline & Cahn-Hilliard (C-H) & \multirow{2}{*}{$\begin{array}{l}\text { simple model for the process of } \\
\text { phase segregation of a binary } \\
\text { alloy at a fixed temperature }\end{array}$} & \multirow{2}{*}{$\begin{array}{l}\text { immiscible } \\
\text { alloys }\end{array}$} & \multirow{2}{*}{$\begin{array}{l}\text { not many } \\
\text { publications }\end{array}$} \\
\hline & Novick-Cohen-Pego & & & \\
\hline & Phase field & simple model for solidification & $\begin{array}{l}\text { tracking of } \\
\text { solidified front }\end{array}$ & $\begin{array}{l}\text { not well developed } \\
\text { for liquid separation }\end{array}$ \\
\hline \multirow{5}{*}{ 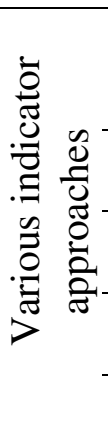 } & $\begin{array}{l}\text { VOF/colour/step } \\
\text { function }\end{array}$ & $\begin{array}{l}\text { well developed for immiscible } \\
\text { fluid, easy to couple with CFD } \\
\text { solver, widely applied }\end{array}$ & $\begin{array}{l}\text { explicit } \\
\text { immiscible } \\
\text { interface }\end{array}$ & $\begin{array}{l}\text { fluid dynamics, } \\
\text { limited accuracy }\end{array}$ \\
\hline & Level set & similar to widely used & \multirow{4}{*}{$\begin{array}{l}\text { explicit } \\
\text { immiscible } \\
\text { interface }\end{array}$} & $\begin{array}{l}\text { reinitialises the } \\
\text { function }\end{array}$ \\
\hline & Front-tracking & $\begin{array}{l}\text { above, but } \\
\text { limited points defined }\end{array}$ & & $\begin{array}{l}\text { fails on complicated } \\
\text { interface }\end{array}$ \\
\hline & Order parameter & $\begin{array}{ll}\text { application, } & \text { coupled C-H and } \\
\text { under } & \mathrm{N}-\mathrm{S} \text { equations }\end{array}$ & & \multirow{2}{*}{ under development } \\
\hline & Segment projection & development segment defined & & \\
\hline \multirow{6}{*}{ 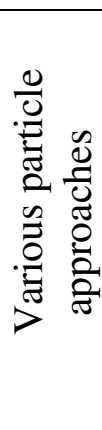 } & PIC: e.g. MAC, etc. & most accurate method & \multirow{6}{*}{$\begin{array}{l}\text { explicit } \\
\text { immiscible } \\
\text { interface }\end{array}$} & $\begin{array}{l}\text { computationally } \\
\text { expensive }\end{array}$ \\
\hline & $\begin{array}{l}\text { lattice gas/Boltzman } \\
\text { lattice-gas }\end{array}$ & $\begin{array}{l}\text { volumeless particle, scale } \\
\text { between N-S and MD }\end{array}$ & & under development \\
\hline & $\begin{array}{l}\text { meshfree-particle } \\
\text { method }\end{array}$ & \multirow{4}{*}{$\begin{array}{l}\text { similar approach coupled with } \\
\text { different schemes }\end{array}$} & & \multirow{4}{*}{$\begin{array}{l}\text { under development, } \\
\text { to be validated }\end{array}$} \\
\hline & particle-MC & & & \\
\hline & $\mathrm{SPH}$ & & & \\
\hline & particle-kinetic & & & \\
\hline \multicolumn{2}{|c|}{ Eulerian model } & \multirow{2}{*}{ mixing, sedimentation, collision } & \multirow{2}{*}{$\begin{array}{l}\text { implicit } \\
\text { interface } \\
\text { mixing }\end{array}$} & \multirow{2}{*}{ macro-scale } \\
\hline \multicolumn{2}{|c|}{ Mixture model } & & & \\
\hline \multicolumn{2}{|c|}{ Moving mesh } & $\begin{array}{l}\text { theoretically developed, limited } \\
\text { application }\end{array}$ & $\begin{array}{l}\text { immiscible } \\
\text { interface }\end{array}$ & limited deformation \\
\hline \multicolumn{2}{|c|}{ Boundary integral } & well developed & $\begin{array}{l}\text { interfacial } \\
\text { dynamics }\end{array}$ & $\begin{array}{l}\text { limited for 3-D } \\
\text { problems }\end{array}$ \\
\hline \multicolumn{2}{|c|}{ Molecular dynamics } & well developed & $\begin{array}{l}\text { biophysics, } \\
\text { chemises }\end{array}$ & atomistic scale \\
\hline \multicolumn{2}{|c|}{$\begin{array}{l}\text { Various } \\
\text { combined/coupled }\end{array}$} & powerful, potential & $\begin{array}{l}\text { immiscible } \\
\text { interface }\end{array}$ & under development \\
\hline
\end{tabular}


The VOF model is a surface-tracking technique applied to a fixed Eulerian mesh. It is designed for two or more immiscible fluids where the position of the interface between the fluids is of interest. In the VOF model, a single set of momentum equations is shared by the fluids, and the volume fraction of each of the fluids in each computational cell is tracked throughout the domain.

Other widely used Euler-Euler multiphase models only track an implicit interface in the macrostructural scale, although the Marangoni and Stokes motions can be included [34] and collisions/coagulations of drops are possibly taken into account by further modification. Therefore, Eulerian multiphase models are only suitable to simulate the hydrodynamic behaviour of droplet/particulate flow in the macrostructural scale without tracking immiscible interfaces between the two-phase system.

As the VOF method provides the possibility of tracking immiscible interfaces that are of interest to the present study, it is employed here for the numerical investigation of the hydrodynamic behaviour of immiscible metallic alloys in the rheomixing process. Details are described in the next section.

The flow of immiscible fluids can be classified into three groups based on the interfacial structure and topographical distributions of the phase, namely segregated flows, transitional and mixed flows and dispersed flows [35]. A large amount of research during the last decades has focused on numerical simulation of flows with immiscible interfaces. Besides the requirements for a good numerical single-phase prediction method, such as conservation, high accuracy, minimal computer storage and calculation, additional requirements are needed for the prediction of interfaces in multi-phase flow, such as:

- the representation of the interface on a discrete grid,

- the movement of the interface with time,

- the treatment of partially filled cells,

- the coupling of the interface conditions with the equations of motion.

There are several immiscible interface modelling methods [36] such as tracking methods (Lagrangian methods), including moving-mesh, front tracking [37,38], boundary integral [39] and particle schemes; capturing methods (Eulerian methods), including volume tracking [40], VOF [41], level set [42], etc. Each of these methods has 
its own advantages and disadvantages. The VOF method provides a simple way of treating topological changes of the interface, such as merging and folding, which may be difficult to handle accurately and directly by other methods. It seems that it is an advantage to use VOF methods in the simulation of drop behaviour in simple shear flow.

VOF is probably the most widely used method for various interface tracking applications. However, it has two problems associated with it:

- VOF is derived for one-dimensional flow and its implementation in higher dimensions is done with the operator splitting approach, so it is limited to structured meshes. Although a possible solution for applying the technique on unstructured meshes has appeared recently [43], its accuracy is still not sufficient for practical applications in engineering.

- The interface tracking formulations can deform the shape of the interface since they depend on grids to maintain the interface curves.

Despite the above limitations, VOF is robust and features in many commercial CFD codes.

In the study of flow composed of two immiscible liquids of different densities and viscosities, one of the greatest difficulties is that the domain of interest contains an unknown free boundary. Therefore, there are three essential features needed to properly model free surfaces:

1. A scheme is needed to describe the shape and location of a surface;

2. An algorithm is required to evolve the shape and location with time;

3. Free - surface boundary conditions must be applied at the surface.

The numerical methods adopted in the present simulations are based on Hirt and Nichols' VOF method [44] coupled with Youngs' piecewise linear interface construction (PLIC) scheme [45], Brackbills' continuum surface force (CSF) model [46], and solved by an algebraic multigrid (AMG) solver [47], as well as the $k-\varepsilon$ turbulence model [48] and the pressure-implicit with splitting of operators (PISO) scheme for pressure-velocity coupling [49]. A brief summary of the PLIC-VOF methodology is provided in what follows. 


\subsubsection{The interface tracking algorithm}

The formulation of the VOF model requires that the convection and diffusion fluxes through the control volume faces be computed and balanced with source terms within the cell itself. The interface will be approximately reconstructed in each cell by a proper interpolating formulation, since interface information is lost when the interface is represented by a volume fraction field. The geometric reconstruction PLIC scheme is employed because of its accuracy and applicability for complex flows, compared to other methods such as the donor-acceptor, Euler explicit, and implicit schemes. A VOF geometric reconstruction scheme is divided into two parts: a reconstruction step and a propagation step. The key part of the reconstruction step is the determination of the orientation of the segment. This is equivalent to the determination of the unit normal vector $\mathbf{n}$ to the segment. Then, the normal vector $\mathbf{n}_{i, j}$ and the volume fraction $C_{i, j}$ uniquely determine a straight line. Once the interface has been reconstructed, its motion by the underlying flow field must be modelled by a suitable algorithm.

Immiscible metallic alloy flows are considered here as multi-phase fluid systems in isothermal state, with different density and viscosity. The domain of interest contains an unknown free boundary, which undergoes severe deformation and separation.

\subsubsection{The volume evolution equations}

The motion of the interface between immiscible liquids of different density and viscosity is defined by the volume fraction function $C$, and the interface is tracked by the following three conditions:

$$
C_{k}(x, y, z, t)=\left\{\begin{array}{l}
0 \text { (outside } k^{\text {th }} \text { fluid) } \\
1 \text { (inside } k^{\text {th }} \text { fluid) } \\
>0,<1 \text { (at the } k^{\text {th }} \text { fluid interface) }
\end{array}\right.
$$

According to the local value of $C_{k}$, appropriate properties and variables are assigned to each control volume within the domain.

The volume fraction function $C_{k}$ is governed by the volume fraction equation:

$$
\frac{\partial C_{k}}{\partial t}+\mathbf{u} \cdot \nabla C_{k}=0
$$

where $\mathbf{u}$ is the flow velocity. 
The flow is governed by a single momentum equation, with the resulting velocity field shared among the phases

$$
\rho\left(\frac{\partial \mathbf{u}}{\partial t}+\mathbf{u} \cdot \nabla \mathbf{u}\right)=-\nabla p+\mu \nabla^{2} \mathbf{u}+\rho \mathbf{g}+\mathbf{F}
$$

where $\mathbf{F}$ stands for body forces, $\mathbf{g}$ for gravity acceleration, and $p$ for pressure. The velocity field is subject to the incompressibility constraint, $\nabla \cdot \mathbf{u}=0$.

In a two-phase system, the properties appearing in the momentum equation are determined by the presence of the component phase in each control volume. The average values of density and viscosity are interpolated by the following formulas

$$
\begin{aligned}
& \rho_{i, j}=\rho_{1}+C_{2}\left(\rho_{2}-\rho_{1}\right) \\
& \mu_{i, j}=\mu_{1}+C_{2}\left(\mu_{2}-\mu_{1}\right)
\end{aligned}
$$

In multi-phase systems, the "onion skin" technique is used [21].

The principal steps of VOF methods are reconstructed interface geometry and time integration algorithms. There are mainly three algorithms (Piecewise constant (SLIC), Piecewise constant stair-stepped (D-A), and Piecewise linear (PLIC)) for the reconstruction interface geometry and two algorithms (one dimensional or operator split and multidimensional or unsplit) for time integration. These include Green-Gauss, volume-average, least-squares, minimization principle, Youngs' gradients, as discussed in [50]. It is noted that a wide, symmetric stencil for $\mathbf{n}_{i, j}$ is necessary for a reasonable estimation of the interface orientation.

The piecewise linear method is different from piecewise constant in that it reconstructs interface lines with a slope, which is given by the interface normal. The interface normal is determined with a multidimensional algorithm that does not rely on the sweep direction.

\subsubsection{Surface force model}

Surface tension along an interface arises as the result of attractive forces between molecules in a fluid. In a droplet surface, the net force is radially inward, and the combined effect of the radial components of forces across the entire spherical surface is to make the surface contract, thereby increasing the pressure on the concave side of the surface. At equilibrium in this situation, the opposing pressure gradient and cohesive 
forces balance to form spherical drops. Surface tension acts to balance the radially inward inter-molecular attractive force with the radially outward pressure gradient across the surface. Surface tension is calculated here by using the CSF scheme [46]. The addition of surface tension to the VOF method is modelled by a source term in the momentum equation. The pressure drop across the surface depends upon the surface tension coefficient $\sigma$,

$$
\Delta p=\sigma\left(\frac{1}{R_{1}}+\frac{1}{R_{2}}\right)
$$

where $R_{1}, R_{2}$ are the two radii, in orthogonal directions, to measure the surface curvature. In the CSF formulation, the surface curvature is computed from local gradients in the surface normal at the interface. The surface normal $\boldsymbol{n}$ is defined by

$$
\boldsymbol{n}_{i, j}=\nabla \mathbf{C}_{\mathbf{i}, \mathbf{j}}
$$

where $C_{i, j}$ is the secondary phase volume fraction.

The curvature $\kappa_{i, j}$ is defined in terms of the divergence of the unit normal $\hat{\boldsymbol{n}}$ :

$$
\kappa=\nabla \cdot \hat{\boldsymbol{n}}=\frac{1}{|\boldsymbol{n}|}\left[\left(\frac{\boldsymbol{n}}{|\boldsymbol{n}|} \cdot \nabla\right)|\boldsymbol{n}|-(\nabla \cdot \boldsymbol{n})\right]
$$

where

$$
\hat{n}=\frac{n}{|n|}
$$

The surface tension can be written in terms of the pressure jump across the interface, which is expressed as a volume force $F$ added to the momentum equation

$$
F_{i, j}=\sigma_{1,2} \kappa_{i, j} \frac{\rho_{i, j} \nabla C}{1 / 2\left(\rho_{1}+\rho_{2}\right)}
$$

where the volume-averaged density $\rho_{i, j}$ is given by Eq.(8).

The CSF model allows for a more accurate discrete representation of surface tension without topological restrictions, and leads to surface tension forces that induce a minimum in the free surface energy configuration. This method has been used by various researchers and is included in most in-house, public and commercial codes, because of its simplicity of implementation. However, the solution quality of PLIC-VOF and CSF is quite sensitive to $\hat{\boldsymbol{n}}=\nabla C /|\nabla C|$, so an accurate estimation of the normal vector often dictates overall accuracy and performance. 


\subsubsection{Summary and Comparison}

Some of the main features of various VOF methods are summarised in Table 3. A comparison of different line techniques for the prediction of the fluid distribution in a cell is illustrated in Fig. 1.

Table 3 Main features of various VOF methods

\begin{tabular}{|c|c|}
\hline Contents & Main Features \\
\hline VOF advantages & $\begin{array}{l}\text { - interface positions are not stored for each time-step } \\
\text { - large surface deformation } \\
\text { - merging and breakup of interfaces } \\
\text { - easy implemented }\end{array}$ \\
\hline $\begin{array}{l}\text { VOF } \\
\text { disadvantages }\end{array}$ & $\begin{array}{ll}\text { - } & \text { interfaces are not exact } \\
\text { - } & \text { reconstruction techniques require many logical operations } \\
\text { - } & \text { resolution-dependent }\end{array}$ \\
\hline SLIC algorithm & $\begin{array}{l}\text { - interface is horizontal or vertical } \\
\text { - assumes that fluid resides on heavy side of interface } \\
\text { - advection: x-sweep, y-sweep }\end{array}$ \\
\hline D-A algorithm & $\begin{array}{l}\text { - interface is horizontal or vertical: piecewise constant, stair } \\
\text { stepped } \\
\text { - derivations of the volume fraction determine whether the } \\
\text { interface is horizontal or vertical } \\
\text { - derivations calculated using fractional volumes averaged over } \\
\text { several cells }\end{array}$ \\
\hline PLIC algorithm & $\begin{array}{l}\text { - interface is piecewise linear } \\
\text { - interface has slope and is fitted within a single cell } \\
\text { - interface slope and fluid position are determined from } \\
\text { inspection of eight neighbouring cells }\end{array}$ \\
\hline
\end{tabular}




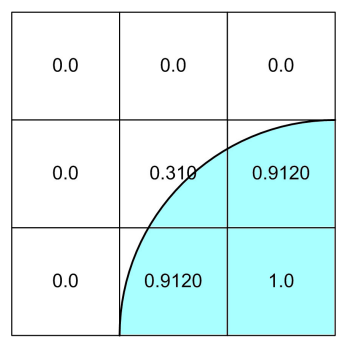

Original Geometry

\begin{tabular}{|l|l|l|}
\hline 0.0 & 0.0 & 0.0 \\
\hline 0.0 & 0.310 & 0.9120 \\
\hline 0.0 & 0.9120 & 1.0 \\
\hline
\end{tabular}

Original Geometry

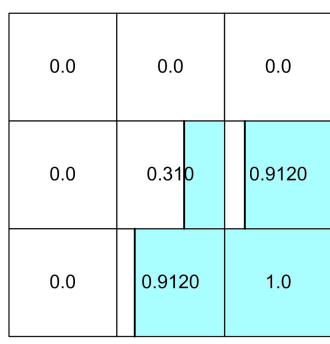

SLIC x-sweep

\begin{tabular}{|c|c|c|}
\hline 0.0 & 0.0 & 0.0 \\
\hline \multirow{2}{*}{0.0} & 0.310 & 0.9120 \\
& & \\
\hline \multirow{2}{*}{0.0} & 0.9120 & 1.0 \\
\hline
\end{tabular}

D-A reconstructed

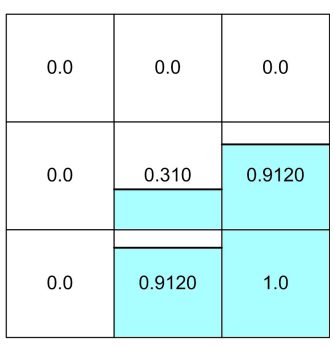

SLIC y-sweep

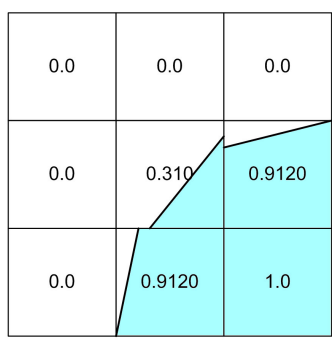

PLIC reconstructed

\section{Fig. 1: Comparison of different line techniques for the prediction of the fluid}

distribution in a cell.

\subsection{Aims of numerical modelling}

The main aim of numerical modelling for studying fundamental mechanisms of rheomixing by the TSE process is to further the understanding of the hydrodynamic behaviour. This is conducted by using CFD techniques incorporating the VOF algorithm, and by investigating immiscible liquid alloys in multi-controlled processes.

The modelling of the basic processes starts with large dispersed drops; thus it begins at capillary numbers much larger than $C a_{\text {crit }}$ (i.e., the critical ratio between the shear stress and the interfacial stress, above which no stable equilibrium drop shape exists). The interfacial stress is overruled by the shear stress (passive interfaces) and simple principles of distributive mixing emerge, where deformation rate and time are interchangeable [51].

\section{Application of PLIC-VOF for immiscible liquid alloy flows}

Numerical simulation seems to have some advantages to investigate immiscible flows at the microscopic level. Therefore, a computer modelling technique based on computational fluid dynamics (CFD) coupled with a PLIC-VOF module is applied to 
solve the problems of decomposition, spatial phase separation and microstructural evolution during the cooling of an alloy through the liquid miscibility gap.

The thermophysical properties of immiscible metallic $\mathrm{Pb}-\mathrm{Zn}$ binary alloys are taken from [52], while phase equilibrium data are taken from [53]. Shear rate is estimated by the equation $\dot{\gamma}=2 n \pi\left(r_{s} / \delta-1\right)$, where $r_{s}$ is the screw radius, $n$ is the screw rotation speed and $\delta$ is the gap between barrel and screw surface [54]. Simulations are conducted on simplified flow fields under shear-induced force, which was implemented as moving boundaries in the computational domain coupled with imposed initial flow field condition. The breakup scale factors $(\mathrm{BSF}) K_{n}^{t} \sim f\left(L_{d}, L_{p}, t\right)\left(L_{d}, L_{p}\right.$ - characteristic length scales of daughter droplet and parent drop, respectively) were proposed by Tang et al. [55] to measure the size of the daughter drop with $t=\xi, t=\psi, t=\zeta$ the time for first daughter drop formation, full breakup up of parent drop, and good distribution of daughter droplets, respectively.

\subsection{Tracking droplet formation}

The results of numerical simulations for one-sided shear-induced flow obtained by using the commercial CFD package FLUENT [56] are shown in Figure 2. The first droplets were formed around $3.6 \mathrm{~ms}$, and the size of the smallest droplet is quite small. The droplet formation occurred in the sheared layer, the wave of the $\mathrm{Pb}$ phase appeared first at the time $2.0 \mathrm{~ms}$, followed by a protruding finger at the time $3.2 \mathrm{~ms}$. The protruding finger then broke up, and droplets formed at the time $3.6 \mathrm{~ms}$

The numerical simulations for two-sided shear flow are shown in Figure 3. In comparison with the one-sided shear condition, there are some different characteristics: drop formation is reached faster than for one-sided shear flow, the droplets are smaller, the morphology of the process is different from one-sided shear flow. Waves appeared suddenly and featured a protruding ball at the time $1.5 \mathrm{~ms}$, and further development of the wave was displayed by protruding fingers. Several small size droplets were initially formed at the time $2.5 \mathrm{~ms}$. Zn phase droplets are entrapped in the $\mathrm{Pb}$ phase; the droplets are either very small or have a long shape, the whole morphology is more complex and the wave is seriously surged. Flows composed of two immiscible liquids and undergoing shearing motion can form fingers as a result of an interfacial instability due primarily to 
the viscosity jump. The jump is in the tangential velocity gradient across the interface, and can be though of as a viscous counterpart of the Kelvin-Helmholtz instability [57]. The process of drop formation displays a good qualitative agreement with hydrodynamic characteristics observed experimentally [58-60].

The simulations ended after the formation of the first few droplets, due to the limitations of computational facilities. For further simulations, a single drop in shearinduced flow is considered in order to examine the microstructural mechanism of drop break-up, which will be discussed in the next section.

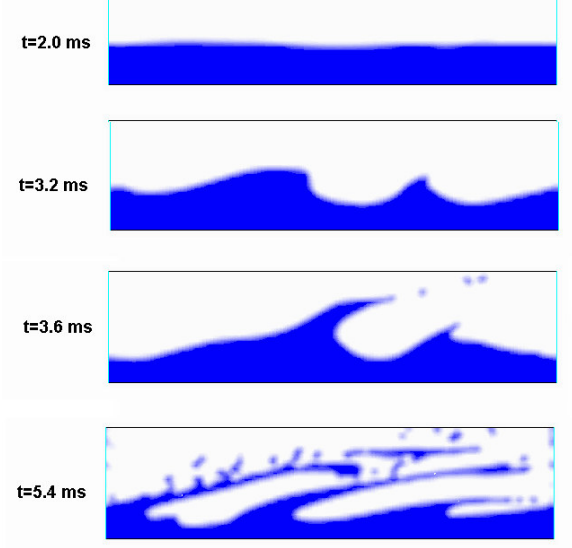

Fig. 2: Numerical evaluation of droplet formation in a 2D one-sided shear-induced flow.

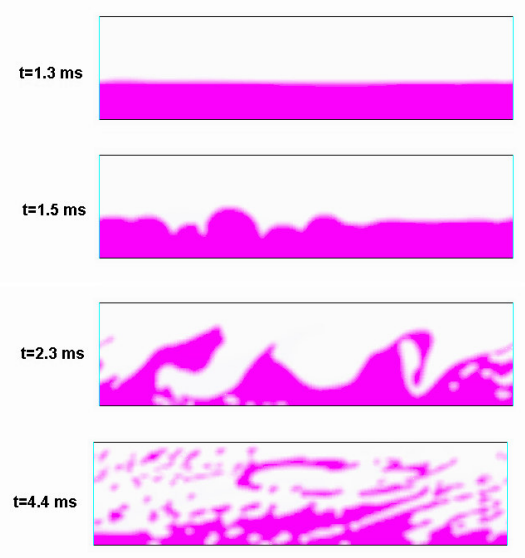

Fig. 3: Numerical evaluation of droplet formation in a 2D two-sided shear-induced flow. 


\subsection{Tracking a drop breakup into droplets}

The initial break-up factor (BSF) $K_{r}^{\xi}$ is defined as the ratio of the capillary number of daughter drop to parent drop, $K_{r}^{\xi}=\mathrm{Ca}_{\mathrm{d}} / \mathrm{Ca}_{\mathrm{p}}=\left(\dot{\gamma} r_{d d} \mu_{m} / \sigma\right) /\left(\dot{\gamma} r_{d} \mu_{m} / \sigma\right)$, in which $r_{d d}$ denotes the daughter drop radius.

The breakup scale factor (BSF) $K_{L}^{\xi}$ is defined as the ratio of the elongative length of the parent drop to the original parent drop diameter, $K_{L}^{\xi}=L_{D} / D_{d}$, at the time the first daughter drop is formed.

For one-sided shear-induced flows, case $1, K_{r}^{\xi}=0.083$, while the ratio of the elongative length to the parent drop radius is $K_{L}^{\xi}=2.167$. For two-sided shear-induced flows, results of the simulations are similar to case 1 .

Daughter drops were born on both sides as shown in Fig. 4, case 2 and case 3. The ratio of the first daughter radius to the parent drop radius $K_{r}^{\xi}$ is equal to 0.0125 , while the ratio of the elongative length to the parent drop diameter $K_{L}^{\xi}$ is equal to 3.417 for case 2. For case 3, the ratio of the first daughter radius to the parent drop radius $K_{r}^{\xi}$ is 0.333 , the ratio of the elongative length to the parent drop diameter $K_{L}^{\xi}$ is 5.542. Case 1 and case 3 need longer for breakup than case 2 .

The effect of viscosity is illustrated in Fig. 5 through case 4 and case 5. The initial breakup factor $K_{r}^{\xi}=0.167, K_{L}^{\xi}=3.833$ for case 4 , and $K_{r}^{\xi}=0.125, K_{L}^{\xi}=2.667$ for case 5 . Case 4 reaches the full breakup much faster than case 5, and the shape of daughter drops in case 5 is longer than in case 4. It appears that the breakup is easier in thin viscous fluids than in thick viscous fluids. This means that the breakup should be completed in the fluid state with low viscosity of the matrix phase in order to obtain fine droplets and short processing time.

During materials processing, drops are in constant movement within the matrix fluid. The material transfer direction is opposite to the direction of screw rotation in the rheomixing process, which means that the shear rate direction is reverse to the direction of fluid velocity. The next simulation was performed by imposing an initial flow field with the material transfer velocity. Results show that drop breakup is much faster in a dynamic flow than in pure shear flow, as illustrated in Fig. 6. The initial breakup factor 
$K_{r}^{\xi}=0.333, K_{L}^{\xi}=2.125$ for case 6 , and $K_{r}^{\xi}=0.4, K_{L}^{\xi}=2$ for case 7 . Turbulence leads to the formation of a round shape as well as speeding up rupturing.

$\mathrm{t}=2.8 \mathrm{~ms}$

Case 1

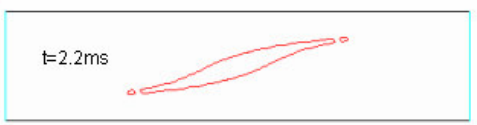

Case 2

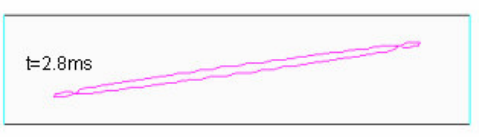

Case 3

Fig. 4: Tracking a drop breakup, grid 128 $\times 32$, domain $16 \times 4$. Cases 1 and 2 are for $\lambda=1, \mathrm{Ca}=3.2$ for one-sided and two-sided shear-induced flow, respectively. Case 3 is for $\mathrm{Ca}=1.17$, initial flow field with $\mathrm{Re}=0$.

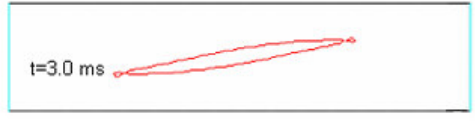

Case 4

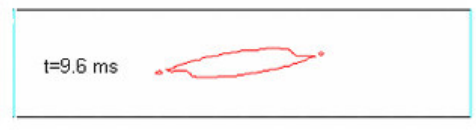

Case 5

Fig. 5: Tracking a drop breakup, grid 128 $\times 32$, domain $16 \times 4, \mathrm{Ca}=2.5$. Case 4 is for $\lambda=1$, case 5 is for $\lambda=0.5$.

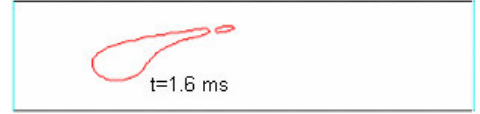

Case 6

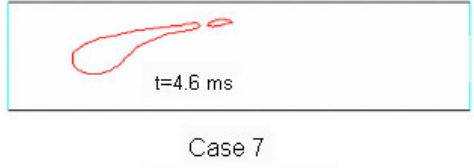

Case 7

Fig. 6: A Pb metallic drop breakup in turbulent flow at the time when the first daughter droplet is formed, grid $128 \times 32$, domain $16 \times 4, \mathrm{Ca}=3.2$; one-sided shearinduced flow. Case 6 is for $\lambda=1$, case 7 is for $\lambda=0.5$.

\subsection{Tracking droplets mixing flow}

The interaction behaviour of daughter droplets involves coalescence and refinement, as illustrated in Figure 7 in which daughter droplets a1, a2 and b1, b2 coalesce to form droplets A12 and B12, and droplets C12 and D12 split into smaller droplets c1, c2 and d1, d2. Moreover, part of droplet D12 was united to droplet E to form a bigger droplet E0.
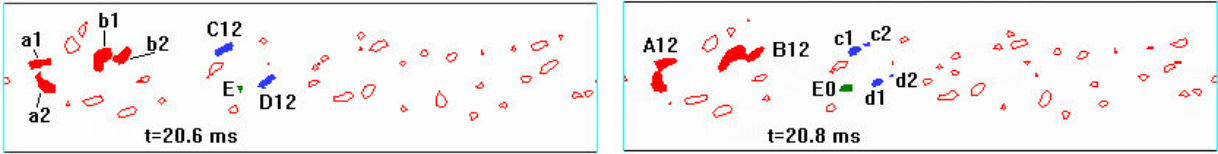

Fig. 7: Illustration of coalescence and refinement of droplets. Daughter droplets are in a mixing stage with further refinement and coalescence occurring simultaneously. 
The breakup scale factor (BSF) $K_{\max }^{t}$ is defined as the ratio of the largest size of daughter drop to parent drop diameter at each time interval, $K_{\max }^{t}=\left.r_{d d \max }\right|_{t=i} / D_{d}, r_{d d \max }$ denotes the largest daughter drop size, $D_{d}$ denotes the diameter of the parent drop.

Similarly, $K_{\min }^{t}$ is defined as the ratio of the smallest size of daughter drop to parent drop diameter at the each time interval, $K_{\min }^{t}=\left.r_{d d \min }\right|_{t=i} / D_{d}, r_{d d m i n}$ denotes the smallest daughter drop size.

A comparison of case 1 and case 2 for the range of $K^{t}$ is shown in Fig. 8 and Fig. 9. It is noted that the range is narrower in one-sided shearing flow (case 1), which is the main flow characteristic of the TSE process. Both $K_{\max }^{t}$ and $K_{\min }^{t}$ are reduced with shearing time, and the first daughter droplet was formed later in the one-sided shearing flow. Case 1 reaches the stage of good distribution of daughter droplets at the time $=24 \mathrm{~ms}$, after which time the $K_{\max }^{t}$ line tends to flaten.

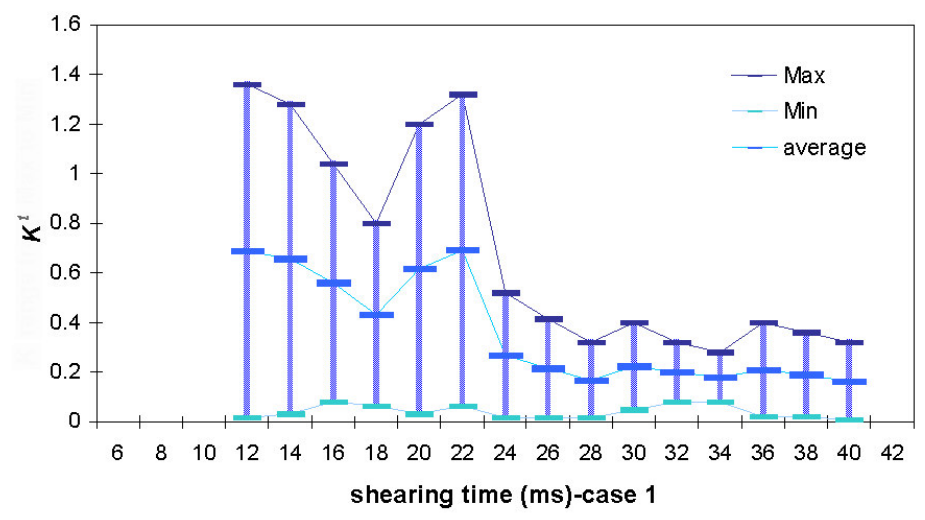

Fig. 8: Schematic illustration of $\boldsymbol{K}^{t}$ range for case 1.

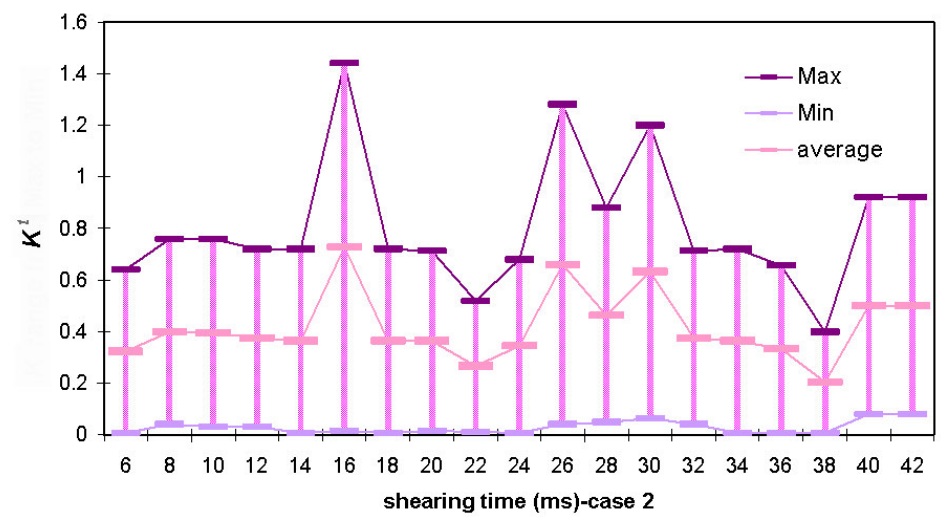

Fig. 9: Schematic illustration of $\boldsymbol{K}^{t}$ range for case 2. 


\subsection{Comparison}

The numerical evolution of droplet dispersion and morphology is compared with an experimental image [61, showing good qualitative agreement.

For a detailed image analysis, a shape factor $K_{\mathrm{k}}$ is defined as $4 \pi$ times the ratio of the droplet area to perimeter square. A perfect circle has a shape factor of 1 , and a line has a shape factor approaching 0 . It is noted that the maximum shape factor $K_{\mathrm{k}}$ is 1.571 for both experiment and simulation. This probably means that the maximum droplet sizes are comparable between experiment and simulation. The present results of numerical simulation show that further numerical predictions can be explored to provide a deeper insight into the microstructure mechanism of the rheomixing process.

Further detailed investigations have been undertaken in order to compare results with experimental and numerical results available in the literature, for the validation of methods and accuracy. Fig.10 shows the velocity vector field for case 8, the flow field inside the drop develops more than one vortex for case 8, and two vortices are clearly visible. Results are in good agreement with the numerical results in [62]. As observed in [63], the two daughter drops contain almost all the volume of the initial drop.

The simulation of case 9 , with properties $\mathrm{Ca}=0.45, \lambda=1$, displays a similar sequence of breakup as in [63], with the two main daughter drops each containing around $50 \%$ of the volume of the initial drop, as shown in Fig. 11.

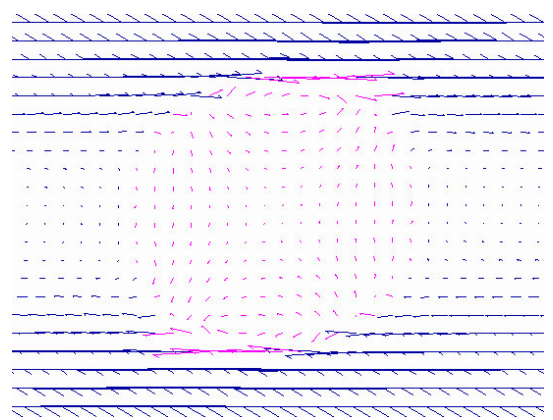

Fig. 10: The velocity vector field of a drop for case 8 , grid $128 \times 32$, domain $16 \times 4$, $\mathrm{Ca}=1.7, \lambda=1$. 


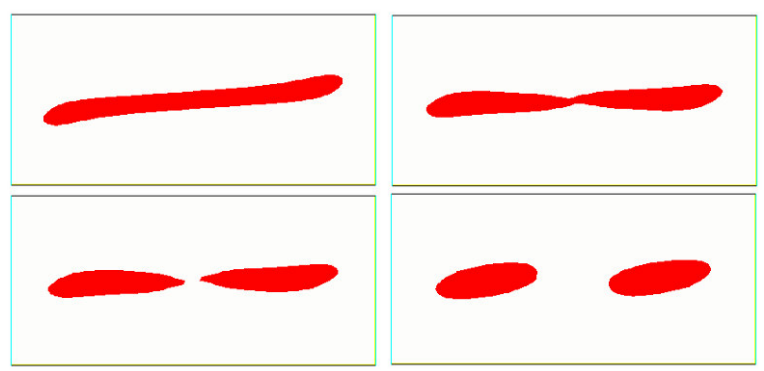

\section{Fig. 11: Drop deformation and breakup for case 9, grid 100×50, domain $1 \times 0.5$, $\mathrm{Ca}=0.45, \lambda=1$.}

\subsection{Discussion}

The present PLIC-VOF approach uses a sheared-field approximation since a single set of mass and momentum equations is adopted. The limitation is that when large velocity differences exist between the phases, the accuracy of the velocities computed near the interface can be adversely affected. A sufficiently fine mesh is required in order to prevent the loss of mass and provide an accurate location. This may be generated adaptively at each time step, but results in increased computing time.

CSF and CSF-based capillary force models treat surface tension as a localised volume force, thus eliminating the need for detailed interface information. They are in principle simple and robust, and require only the phase indicator $C$ to be determined. However, both are known to induce 'spurious currents' near the interface, because once discretized, the exact momentum jump condition at the interface is not always properly preserved, i.e. pressure and viscous stress forces do not balance the capillary forces. This is partly due to the lack of precision in solving the curvature, but it also results from the way the surface term is discretized in the momentum equation.

If the accuracy of the VOF method can be reached by a reasonably fine grid to ensure that the line of interface of the immiscible fluids in each cell is as close as possible to a straight line, the loss of mass will be minimised and the interface morphology will approximate the real one. For capturing a droplet with diameter of $0.02 \mathrm{~mm}$, as shown in Fig. 12, there is still loss of mass with a grid size of 0.0025 .

The diameter of a drop should be meshed by a grid of the order of 32 cells or more for VOF methods. For instance, for drop deformation in cases 1 to 7 , maximum grid size $=$ $\mathrm{r}_{\mathrm{d}} / 16=1 / 16=0.0625 \mathrm{~mm}$, and for capturing a droplet with the diameter of $0.02 \mathrm{~mm}$, grid 
size $=r_{d} / 16=0.000625 \mathrm{~mm}$. However, the actual grid size for the deformation in cases 1 to 7 is $r_{d} / 8=0.125 \mathrm{~mm}$. The grids are two times larger than desired for the deformation evolution and 200 times larger than desired for capturing a droplet of $0.02 \mathrm{~mm}$ diameter in cases 1 to 7 . Hence, there is a loss of mass, especially for the breakup evolution, due to the limitation of computational resources and numerical round-off error, so that the numerical investigation should focus on qualitative aspects and maximum size of droplets. A possible improvement is to apply adaptive meshing at certain time steps to refine grids around the immiscible droplet interfaces.

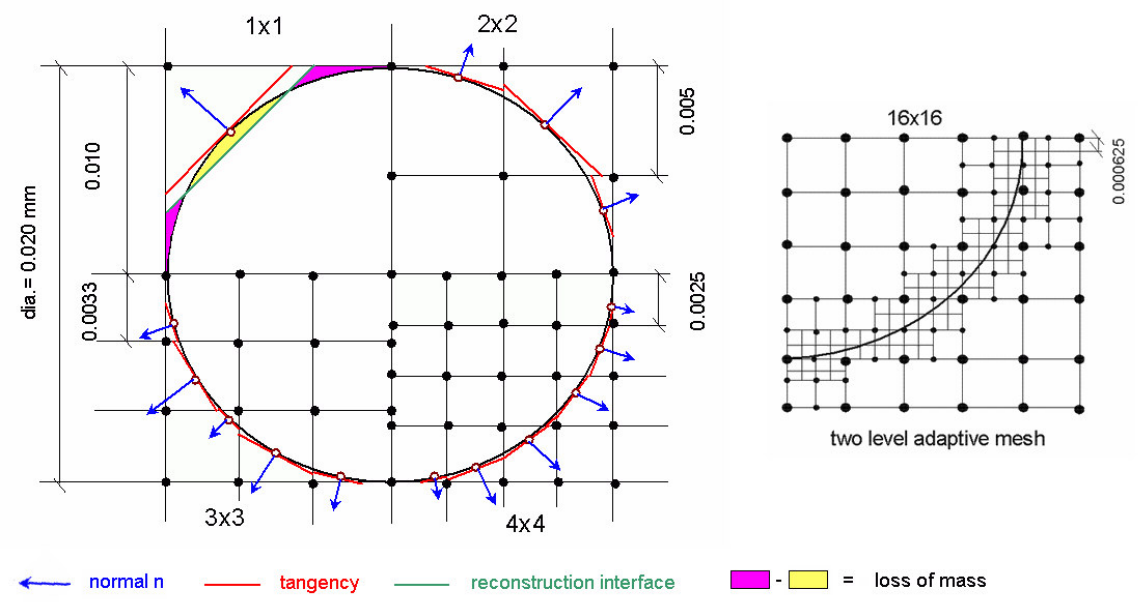

\section{Fig. 12: Mesh comparison for different grid sizes (left) and two-level adaptive mesh approach (right).}

\section{Conclusions}

Numerical simulations have been conducted using FLUENT [56] as simplified problems through the VOF method with the PLIC scheme, AMG approach and $k-\varepsilon$ turbulence model. The metallic drop deformation and rupturing, the essential microscopic mechanisms of the rheomixing process, were investigated to increase our understanding of the basic behaviour of immiscible metallic drops in a prototypical rheomixing process. The results show that numerical methods are capable of simulating the rheological behaviour of an immiscible $\mathrm{Zn}-\mathrm{Pb}$ binary alloy in shear-induced mixing processes, which reflect the fundamental flow features of twin-screw rheomixing process. 


\section{Acknowledgements}

We are grateful to researchers in the CFD groups at the School of Aerospace, Mechanical \& Manufacturing Engineering, RMIT University, Australia and at the School of Engineering and Design, Brunel University, UK, for helpful discussions on numerical approaches and the TSE rheomixing casting process.

\section{References}

[1] S. Ji, Z. Fan, M.J. Bevis, 2001 Semi-solid processing of engineering alloys by a twin-screw rheomoulding process, Mat. Sci. Eng. A 299 (2001) 210-217.

[2] N.P. Cheremisinoff, Polymer Mixing and Extrusion Technology, Marcel Dekker, New York, 1987.

[3] C. Rauwendaal, Mixing in Polymer Processing, Marcel Dekker, New York, 1991.

[4] L.L. Lacy, G. Otto, The behaviour of immiscible liquids in space, AIAA/ASME paper 74-668, Boston Mass, 1974; cited in: J. Jia, J.Z. Zhao, J.J. Guo, Y. Liu, Immiscible Alloys and Fabrication Technology, HIT, Harbin (China), 2002.

[5] R. Jansen, P.R. Sahm, Solidification under microgravity, Mat. Sci. Eng. 65 (1984) 199-212.

[6] J.Z. Zhao, S. Drees, L. Ratke, Strip casting of Al-Pb alloys -- a numerical analysis, Mat. Sci. Eng. A 282 (2000) 262-269.

[7] J. F. Agassant, A. Poitou, A kinematic approach to distributive mixing, in: Mixing and Compounding of Polymers, , I. Manas-Zloczower and Z. Tadmor (eds.), Hanser, New York, 1994.

[8] M.L. Booy, Isothermal flow of viscous liquids in co-rotating twin screw devices, Polym. Eng. Sci. 20 (1980) 1220

[9] P.G. Andersen, Mixing practices in corotating twin-screw extruders in: Mixing and Compounding of Polymers, I. Manas-Zloczower and Z. Tadmor (eds.), Hanser, New York, 1994.

[10] Z. Fan, Semisolid metal processing, Int. Mater. Rev. 47 (2002)49-85.

[11] J.L. White, Twin Screw Extrusion: Technology and Principles, Hanser, Munich, 1990.

[12] L. Ratke, S. Diefenbach, Liquid immiscible alloys, Mat. Sci. Eng. RI5 (1995) 263-347

[13] M. Smoluchowiski, Drei vortrage uber diffusion, Phys. Z. 17 (1916) 557, cited in [12].

[14] J. Rogers, R. Davis, Modelling of collision and coalescence of droplets during microgravity processing of Zn-bi immiscible alloys, Metal Trans. A 21 (1990) 59-64.

[15] L. Ratke, H. Fischmeister, A. Kneissl, Monotectic solidification of $\mathrm{Cu}-\mathrm{Pb}$ alloys, in: $6^{\text {th }}$ European Symposium on Material Science under Microgravity Condition, ESA SP-256, 161-165, 1987.

[16] L. Ratke, H. Frschmerister, A. Kneissl, Measurement of diffusion coefficient of copper in liquid lead in microgravity in: $7^{\text {th }}$ European Symposium on Material Science under Microgravity Condition, ESA SP-295, 135-139, 1989

[17] L. Alkemper, L. Ratke, Concurrent nucleation, growth and sedimentation during solidification of AlBi alloys, Z Metall 85 (1994) 365-371.

[18] T. Carberg, H. Fredriksson, The influence of microgravity on the structure of $\mathrm{Zn}-\mathrm{Bi}$ immiscible alloys, Metal Trans. 11a (1982) 1665-1676.

[19] A. Bergman, H. Fredriksson, A study of the coalescence process inside the miscibility gap in Zn-Bi alloys, in: Material Processing in the Reduced Gravity Environment of Space, G.E. Rindones (ed.), Elsevier, 563-577, 1982.

[20] H. Ahlborn, H. Neumann, H.J. Schott, Segregation behaviour of rapidly cooled monotectic Al-In and Al-Pb alloys, Z Metall 84 (1993) 748-754.

[21] J.Z. Zhao, L. Ratke, Repeated nucleation of immiscible of minority phase droplets induced by drop motion, Scripta Mater. 39 (1998) 181-188.

[22] J.Z. Zhao, L. Ratke, Modelling of rapid unidirectional solidification of hypermonotectic alloys, Mater. Lett. 38 (1999) 235-238.

[23] Y. Efendiev, M.R. Zachariah, Hybrid Monte Carlo method for simulation of two-component aerosol coagulation and phase segregation, J. Colloid Interf. Sci. 249 (2002) 30-43.

[24] L. Ratke, S. Diefenbach, B. Prinz, H. Ahlborn, Marangoni motion of a hypermonotectic alloynumerical simulation for the D2 experiment IHF04, in: $18^{\text {th }}$ European Symposium on Material Science under Microgravity Condition, ESA SP-333, 495-501, 1992. 
[25] S. Diefenbach, PhD Thesis, Ruhr-Universität, 1994; cited by [12] p325.

[26] P.V. Coveney, K.E. Novik, Computer simulations of domain growth and phase separation in twodimensional binary immiscible fluids using dissipative particle dynamics, Phys. Rev. E 54 (1996) 5134-5141.

[27] D.H. Rothman, S. Zaleski, Lattice-Gas Cellular Automata: Simple Models of Complex Hydrodynamics, Cambridge University Press, 1997.

[28] G. Leptoukh, B. Strickland, Ch. Roland, Phase separation in two-dimensional fluid mixtures, Phys. Rev. Lett. 74 (1995) 3636-3639.

[29] G. Peng, F. Qiu, V.V. Ginzburg, D. Jasnow, A.C. Balazs, Forming supramolecular networks from nanoscale rods in binary, phase-separating mixtures, Science 288 (2000) 1802-1804.

[30] K.E. Novik, P.V. Coveney, Using dissipative particle dynamics to model binary immiscible fluids, Int. J. Mod. Phys. C 8 (1997) 909-915.

[31] P.C. Fife, Models for phase separation and their mathematics, J. Differ. Equations 48 (2000) 1-26.

[32] T. Kupper, N. Masbaum, Simulation of particle growth and Ostwald ripening via the Cahn-Hillard equation, Acta Metal Mater. 42 (1994) 1847-1857.

[33] L. Ratke, P.W. Voorhees, Growth and Coarsening, Ostwald Ripening in Matrials Science, SpringerVerlag, Heidelberg, 2002.

[34] M. Wu, A. Ludwig, L. Ratke, Modelling the solidification of hypermonotectic alloys, Model. Simul Mater. Sci. Eng. 11 (2003) 755-769.

[35] M. Ishii, Thermo-fluid dynamic theory of two-phase flow, 1975; cited by O. Ubbink, Numerical prediction of two fluid systems with sharp interfaces, PhD Thesis, The University of London, 1997.

[36] R. Scardovelli, S. Zaleski, Direct numerical simulation of free surface and interfacial flow, Annu. Rev. Fluid Mech. 31 (1999) 567-603.

[37] R.W. Yeung, Numerical methods in free-surface flows, Annu. Rev. Fluid Mech. 14 (1982) 395-442.

[38] S.O. Unverdi, G. Tryggyason, A front-tracking method for viscous, incompressible, multi-fluid flows, J. Comput. Phys. 100 (1992) 25.

[39] V. Cristini, J. Blawzdziewicz, M. Loewenberg, Drop breakup in three-dimensional viscous flows, Phys. Fluids 10 (1998) 1781-1783.

[40] B.D. Nichols, C.W. Hirt, R.S. Hotchkiss, SOLA-VOF: A solution algorithm for transient fluid flow with multiple free boundaries, Los Alamos Scientific Laboratory Report, LA-8355,1980.

[41] C.W. Hirt, B.D. Nichols, A computational method for free surface hydrodynamics, J. Press. Vess-T ASME 103 (1981) 136.

[42] S. Osher, J.A. Sethian, Fronts propagating with curvature-dependent speed: algorithms based on Hamilton-Jacobi formulations, J. Comput. Phys. 79 (1988) 12.

[43] Y. Zhao, H.H. Tan, B. Zhang, A high-resolution characteristics-based implicit dual time-stepping VOF method to free surface flow simulation on unstructured grids, J. Comput. Phys. 183 (2002) 233273.

[44] C.W. Hirt, B.D. Nichols, Volume of fluid VOF for the dynamics of free boundaries, J. Comput. Phys. 39 (1981) 201.

[45] D.L. Youngs, Time-dependent multi-material flow with large fluid distortion, in: Numerical Methods for Fluid Dynamics, K.W. Morton and M.J. Baines (eds.), Academic Press, New York, 273-285, 1982.

[46] J.U. Brackbill, D.B. Kothe, C. Zemach, A continuum method for modeling surface tension, J. Comput. Phys. 100 (1992) 335-354.

[47] K. Stüben, A review of algebraic multigrid, J. Comput. Appl. Math. 128 (2001) 281-309.

[48] B.E. Launder, D.B. Spalding, The numerical computation of turbulent flows, Comput. Meth. App. Mech. Engng. 3 (1974) 267-289.

[49] R.I. Issa, A.D. Gosman, A.P. Watkins, The computation of compressible and incompressible recirculating flows by a non-iterative implicit scheme, J. Comput. Phys. 93 (1991) 388-410.

[50] D.B. Kothe, W.J. Rider, S.J. Mosso, J.S. Brock, Volume tracking of interface having surface tension in two and three dimensions, in: $34^{\text {th }}$ Aerospace Science Meeting and Exhibition, AIAA960859,1996 .

[51] H.E.H. Meijer, M.H. Janssen, Mixing of immiscible liquids, in: Mixing and Compounding of Polymers, I. Manas-Zloczower and Z. Tadmor (eds.), Hanser, New York, 52-85, 1994.

[52] T. Iida, R.I.L. Guthrie, The Physical Properties of Liquid Metals, Oxford University Press, New York, 1988. 
[53] R. Hultgren, R.L. Orr, P.D. Anderson, K.K. Kelley, Selected Values of the Thermoldynamic Properties of Binary Alloys, Metals Park, ASM, 1973.

[54] C. Rauwendaal, Polymer Extrusion, $3^{\text {rd }}$ rev. ed, Hanser, New York, 1994.

[55] H. Tang, L.C. Wrobel, Z. Fan, Tracking of immiscible interfaces in multiple-material mixing processes, Computational Materials Science 29 (2004) 103-118.

[56] FLUENT Documentation (User's Guide, UDF Manual, Text Command List) Version 5, Fluent Inc, Lebanon, NH, 1998.

[57] J. Li, Y.Y. Renardy, M. Renardy, A numerical steady of periodic distributions on two-layer Couette flow, Physics of Fluids 10 (1998) 3056-3071.

[58] D.D. Joseph, K. Nguyen, G. Beavers, Non-uniqueness and stability of the configuration of flow of immiscible fluids with different viscosities, J. Fluid Mech. 141 (1984) 319-345.

[59] P. Barthelet, F. Charru, Benjamin-Feir and Eckhaus instabilities with Galilean invariance: the case of interfacial waves in viscous shear flows, Eur. J. Mech. B/Fluids 17 (1998) 1-18.

[60] F. Charru, P. Barthelet, Secondary instabilities of interfacial waves due to coupling with a long wave in a two-layer Couette flow, Physica D 125 (1999) 311-324.

[61] X. Fang, Z. Fan, S. Ji, Y. Hu, Processing of immiscible alloys by a twin-screw rheomixing process, in: Proceedings of the $7^{\text {th }}$ Advanced Semisolid Processing of Alloys and Composites, Y. Tsutsui, M. Kiuchi and K. Ichikawa (eds.), Tsukuba, Japan, 695-700, 2002.

[62] J. Li, Y.Y. Renardy, Shear-induced rupturing of a viscous drop in Bingham liquid, J. Non-Newton. Fluid Mech. 95 (2000) 235-251.

[63] C. R. Mark, Drop breakup and deformation in sudden onset strong flows, PhD Thesis, University of Maryland at College Park, 1998. 